\title{
Algorithms for Real-Time FastHARP Cardiac Function Analysis
}

\author{
Khaled Z. Abd-Elmoniem and Jerry Prince \\ Electrical and Computer Engineering, Johns Hopkins University \\ Baltimore, MD 21218, USA \\ \{khaled,prince\} @jhu.edu \\ http://iacl.ece.jhu.edu/
}

\begin{abstract}
FastHARP is a magnetic resonance pulse sequence that can acquire tagged cardiac images at $40 \mathrm{~ms}$ per frame. Data from just two heartbeats can be used to compute in-plane quantities using the harmonic phase (HARP) method for describing myocardial deformation, such as circumferential and radial strain. Although HARP computations have previously been coded to keep pace with the FastHARP pulse sequence, further computational speed improvements are necessary in order to achieve either smoother strain images using viewsharing concepts or more tracked points for more detailed strain analysis. In this work, we present faster processing routines that allow improved temporal resolution and more detailed real-time tracking and analysis. With this code, HARP strain computations can now be computed and displayed in real-time after a one heartbeat delay with a temporal interval of $20 \mathrm{~ms}$.
\end{abstract}

\section{Introduction}

Left ventricular function, especially when evaluated during exercise or dobutamineinduced stress can reveal valuable information about myocardial health. The ability to rapidly detect the onset of an ischemic event is crucial to patient safety during a cardiac stress exam. Detecting ischemia is particularly problematic in magnetic resonance (MR) imaging, where the EKG is less reliable, and real-time imaging has heretofore been limited to anatomical imaging. Real-time visualization of regional cardiac function using MR tagging [1] has only recently become possible using a fast harmonic phase (FastHARP) pulse sequence [7]. FastHARP is an MR imaging pulse sequence that allows real-time acquisition of tagged cardiac images, typically shortaxis slices of the left ventricle. FastHARP enables the acquisition of 7-20 HARP images in a single heartbeat for a given tag orientation. In the next heartbeat, an analogous sequence is acquired with tags oriented in the orthogonal direction. Data from two heartbeats is therefore sufficient to compute in-plane quantities describing myocardial deformation, such as circumferential and radial strain.

Previously, to compute radial or circumferential strain, HARP software required about one second per frame, which is not adequate for real-time visualization [2]. Recently, we developed a system for real-time cardiac strain visualization using the FastHARP imaging sequence. The system integrates FastHARP acquisition in parallel 
with a rapid strain computation and visualization tool $[9,10]$. In this system, HARP strain computations are carried out in real-time after a one heartbeat delay. In addition to the computation and display of strain maps, it is also possible to track the time profile of strain of one or more points in the myocardium.

In this paper, we present two real-time algorithms which further reduce image reconstruction time and, thus, the overall strain computation time. This reduction, when combined with view-sharing, doubles the temporal resolution, allows phase correction routines to be incorporated, and permits more comprehensive strain analyses to be performed in real-time.

\section{Background}

\subsection{FastHARP}

Acquisition. HARP provides automatic post-processing methods for cardiac strain analysis [2,4,5]. FastHARP [7] is an MR imaging pulse sequence designed for realtime tagged cardiac imaging. It acquires only that portion of $\mathrm{k}$-space that is critical to HARP processing, as illustrated in Fig. 1.. FastHARP uses 1-1 SPAMM tags at enddiastole, and acquires only $32 \times 32$ points over a square region centered at a selected harmonic peak in k-space. These k-space points are acquired using a multi-shot, echoplanar pulse sequence comprising 8 shots with 4 echoes each (see Fig. 1c.). This can be accomplished in only about $45 \mathrm{~ms}$ allowing the acquisition of 7-20 HARP images in a single heartbeat for a given tag orientation. In the next heartbeat, a sequence of HARP images is acquired in the same way, but with the tags oriented in the orthogonal direction. The phase of the image created from the off-origin spectral peak is directly related to the tissue motion, which is the principle of HARP automatic processing of tagged MR images (see Fig. 1f.). Spatial derivatives of harmonic phase are computed at each pixel to obtain strain measures.

Synthetic Tagging. Harmonic magnitude images are devoid of tags, and therefore contain little useful motion information. Harmonic phase images contain most of the motion information, but the phase wrapping artifacts and lack of magnitude information makes them difficult to visualize. Synthetic tagging is a fast technique for providing balanced visualization of both anatomical and tagging [6]. Synthetic tagged images can be generated from harmonic images by periodically replicating in Fourier space the single imaged spectral peak. A fast, convenient, and mathematically rigorous way to do this spectral replication is to use the following Fourier series expansion

$$
I_{\text {synth }}=c_{0} D(\mathbf{p}, t)+c_{1} D(\mathbf{p}, t) \sin (\varphi(\mathbf{p}, t))+c_{2} D(\mathbf{p}, t) \cos (2 \varphi(\mathbf{p}, t))+c_{3} D(\mathbf{p}, t) \sin (3 \varphi(\mathbf{p}, t)) .
$$

Here, $c_{0}, c_{1}, c_{2}$, and $c_{3}$ are a set of coefficients that determine the sharpness of the tags. The vector $\mathbf{p}(x, y)$ is the spatial position of a pixel in the image plane and $t$ is the time that has elapsed after tag application. The magnitude image $D(\mathbf{p}, t)$ is as in Fig. 1e and the phase image $\varphi(\mathbf{p}, t)$ is as in fig. 1f. The resulting image comprises the magnitude image superposed with crisp tag lines that appear like conventional higher-order SPAMM tags and move like them as well (see Fig. 1g.). 


\subsection{HARP Strain Analysis}

From two harmonic phase images $\mathrm{a}_{1}$ and $\mathrm{a}_{2}$ with mutually perpendicular tag directions with tag frequencies $\omega_{1}$ and $\omega_{2}$ respectively, the Eulerian strain (difference in displacement between adjacent parts of the myocardium) is computed as follows

$$
\varepsilon_{a}(\mathbf{p}, t ; \mathbf{n})=\frac{\left\|\left[\nabla^{*} \mathbf{a}(\mathbf{p}, t)\right]^{-1} \mathbf{W}^{-T} \mathbf{n}\right\|}{\|\mathbf{n}\|}-1,
$$

where

$$
\mathbf{a}(\mathbf{p}, t) \equiv\left[\begin{array}{l}
\mathrm{a}_{1}(\mathbf{p}, t) \\
\mathrm{a}_{2}(\mathbf{p}, t)
\end{array}\right], \mathbf{W} \equiv\left[\begin{array}{l}
\omega_{1} \\
\omega_{2}
\end{array}\right]^{T},
$$

$\mathbf{n}$ is the direction along which the Eulerian strain is computed and the superscript $T$ stands for the transpose of the vector or matrix (see Fig. 1h.).

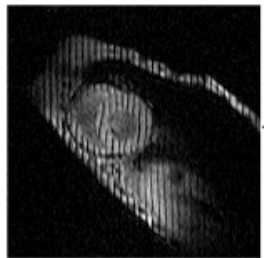

(a)

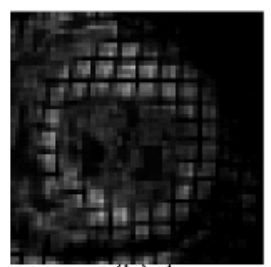

(h)

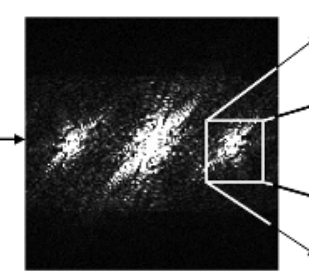

(b)

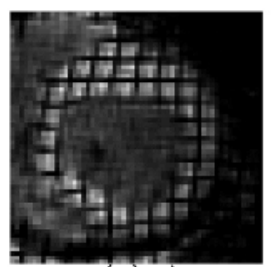

(g)

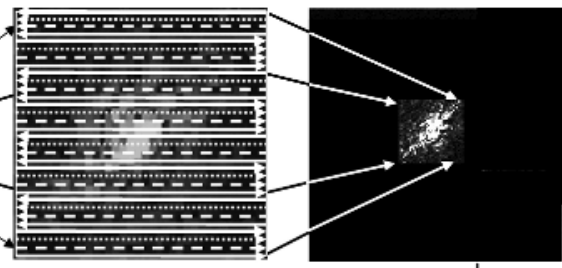

(c)

(d)

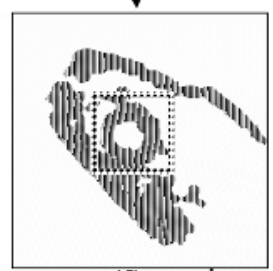

(f)

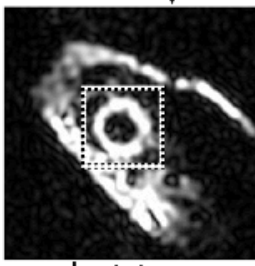

(e)

Fig. 1. (a) Tagged MRI. (b) k-space image. (c) Acquiring the first harmonic peak of tagged image using fastHARP, $S\left[k_{x}, k_{y}\right]$ using 8 shots with 4 echoes each. (d) Zero padding. (e) Magnitude image $D(\mathbf{p}, t)$ showing a typical region of interest. (f) Phase image $\varphi(\mathbf{p}, t)$. (g) ROI with synthetic tagging superimposed. (h) Eulerian strain map

\section{Methods}

In Fig. 1d., zero-padding in the Fourier domain was used in order to obtain a spatial image with a reasonable pixel resolution Fig. 1(e and f). The resulting image is also cropped to retain a (typically) much smaller region of interest (ROI), as shown in 
Fig 1(e and $\mathrm{f}$ ). This overall process, however, requires more than $30 \mathrm{~ms}$ of computation time, which is a large fraction of the $45 \mathrm{~ms}$ data acquisition cycle in FastHARP.

In this work, we investigated two algorithms to reconstruct an ROI from the raw k-space data $S\left[k_{x}, k_{y}\right]$ as shown in Fig. 1c: 1) the chirp Fourier transform (CFT) and 2) B-spline interpolation (BSI). CFT and BSI share the advantage that most of the computations can be made offline, saving time in comparison to the present IFT approach. Also, both algorithms are separable, so that 2D signals can be processed successively in the rows and then the columns. Therefore, in the following analysis, we present the $1 \mathrm{D}$ case, which is trivially extended to handle our actual $2 \mathrm{D}$ problem.

\subsection{Chirp Fourier Transform (CFT)}

Given a $K$-point sequence $S[k], 0 \leq k<K$ as input, the conventional inverse Fourier Transform (FT) provides a spatial pixel resolution proportional to $1 / K$. If this resolution is not sufficient in a given application, zero padding the input sequence is used to get better resolution. This is an expensive operation if the required resolution is much higher than the original one. Moreover, in our case we are interested in only a small ROI, so computing the whole FOV is wasteful. The chirp Fourier Transform (CFT) accomplishes this task with much lower cost [12].

Theory of CFT. Given the 1D sequence $S[k]$ sampled in the frequency domain, representing a row or a column of the acquired k-space data $S\left[k_{x}, k_{y}\right]$, we wish to compute the signal $s(x)$ in the spatial domain

$$
s(x)=\sum_{k=0}^{K-1} S[k] e^{j\left(2 \pi \frac{k}{K}\right) x},
$$

where $\Delta x$ is the desired spatial resolution, and

$$
x(n)=x_{0}+n \Delta x, n \in Z, 0 \leq n<N .
$$

Define $W=e^{j\left(2 \pi \frac{k}{K}\right) \Delta x}$. (3) can be rewritten as

$$
s(x(n))=\sum_{k=0}^{K-1} S[k] e^{j\left(2 \pi \frac{k}{K}\right) x_{0}} W^{n k} .
$$

Equivalently,

$$
s(x(n))=W^{0.5 n^{2}} \sum_{k=0}^{K-1} S[k] e^{j\left(2 \pi \frac{k}{K}\right) x_{0}} W^{0.5 k^{2}} W^{-0.5(n-k)^{2}} .
$$

Upon defining the following sequences

$$
H[k]=W^{-0.5 k^{2}} \quad, \quad F[k]=e^{j\left(2 \pi \frac{k}{K} x_{0}\right)} W^{0.5 k^{2}} \quad, \quad l[n]=W^{0.5 n^{2}},
$$

then (6) takes the form

$$
G[k]=S[k] \times F[k] .
$$




$$
s(x(n))=l[n] \times\{G * H\}[n]
$$

The convolution is done using fast inverse Fourier transform (IFFT) as

$$
s(x(n))=l[n] \times F F T\{\operatorname{IFFT}\{G\} \times \operatorname{IFFT}\{H\}\}[n] .
$$

We refer the reader to [12] for detailed implementation.

Application. Because of the separability property of the Fourier transform, we can apply (8) followed by (10) on the rows and then the columns of the raw acquired data $S\left[k_{x}, k_{y}\right]$. Moreover, $H[k], F[k], \operatorname{IFFT}\{H[k]\}$, and $l[n]$ can all be computed offline, which significantly reduces the online processing time.

\subsection{B-Spline Interpolation (BSI)}

The second approach to rapidly obtain the spatial ROI to first transform the acquired $32 \times 32$ raw k-space data points $S\left[k_{x}, k_{y}\right]$ into the spatial domain as an image $s[n, m]$, $0 \leq n, m<32$. Then, after representing the spatial data as a continuous function $s(x, y)$, the discrete ROI image is simply sampled from $s(x, y)$. Here, we use B-spline functions [11] as the basis for fitting the continuous function.

Theory of BSI. Like CFT, BSI is also a separable algorithm [11]. Given 1D input signal values $s[n], 0 \leq n<N$, which is found in our case by inverse transforming the raw Fourier data, the parameterized continuous signal model $s(x)$ can be approximated as a spline, which is a piecewise polynomial with pieces that are smoothly connected together. The joining points of the polynomial are called knots. For a spline of degree $k$, each segment is itself a polynomial of degree $k$. With the requirement that the spline and its derivates up to order $(k-1)$ are continuous, there is only one degree of freedom per segment. Therefore, this spline is uniquely characterized in terms of a B-spline expansion

$$
s(x)=\sum_{n=0}^{N-1} c[n] \beta^{k}(x-n),
$$

which involves the integer shifts of the central B-spline of degree $k$ denoted by $\beta^{\mathrm{k}}(x)$. The parameters of the model are the B-spline coefficients $c[n]$.

When $k=3$, The B-splines are called cubic splines. In this case, the basis function takes the form

$$
\beta^{3}(x)=\left\{\begin{array}{cc}
\frac{2}{3}-|x|^{2}+\frac{|x|^{3}}{2}, & 0 \leq x<1 \\
\frac{(2-|x|)^{3}}{6}, & 1 \leq x<2 \\
0, & 2 \leq x,
\end{array}\right.
$$

The only work is to determine the B-spline coefficients $c[n]$ such that the function $s(x)$ goes through the data points exactly. 
Algorithm. A recursive causal and anti-causal filtering scheme is employed to obtain the coefficients $c[n][11]$. With $\mathrm{z}_{1}=-2+\sqrt{3}$ and $c^{-}[n]=c[n] / 6$, the following algorithm is used

$$
\begin{array}{ll}
c^{+}[n]=s[n]+z 1 c^{+}[n-1], & (n=1, \ldots, N-1) \\
c^{-}[n]=z_{1}\left(c^{-}[n+1]-c^{+}[n]\right), & (n=N-2, \ldots, 0)
\end{array}
$$

with the following initial conditions

$$
\begin{aligned}
& c^{+}[0]=\frac{1}{1-z_{1}{ }^{2 N-2}} \sum_{n=0}^{2 N-3} s[n] z_{1}{ }^{n} . \\
& c^{-}[N-1]=\frac{z_{1}}{\left(1-z_{1}^{2}\right)}\left(c^{+}[N-1]+z_{1} c^{+}[N-2]\right) .
\end{aligned}
$$

Application. With 2D image point values $s[n, m], 0 \leq n<N, 0 \leq m<M$ the algorithm above can be applied successively along the rows and columns of the image. The spline model for a particular location $(x, y)$ in the image is given by

$$
s(x, y)=\sum_{m=0}^{M-1} \sum_{n=0}^{N-1} c[n, m] \beta^{3}(x-n) \beta^{3}(y-m) .
$$

By this scheme, a higher resolution image of ROI is obtained by sampling the continuous image function in (15). The algorithm has two parts; the first part is the offline procedure in which the function $\beta^{3}(x-k) \beta^{3}(y-l)$ centered at $(k, l) \quad 0 \leq k, l<32$ is evaluated at all the points $(x, y)$ in the ROI. The online part consists of the inverse FT of the off-origin harmonic peak $S\left[k_{x}, k_{y}\right]$ shown in Fig. 1c, the evaluation of the spline coefficients from (13) and (14), and finally evaluating $s(x, y)$ from (15).

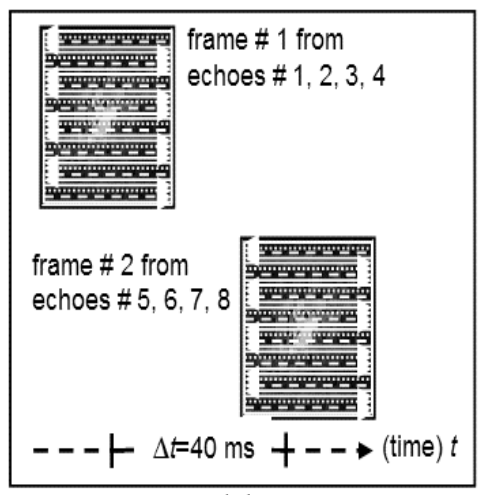

(a)

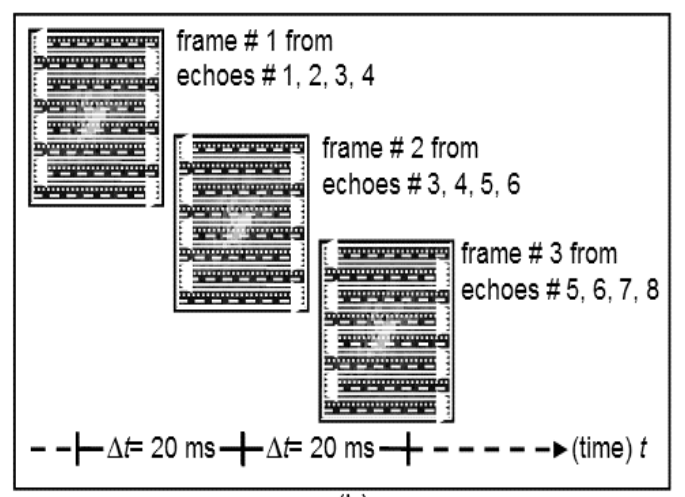

(b)

Fig. 2. FastHARP with view sharing. (a) Conventional acquisition, an image is constructed every $40 \mathrm{~ms}$. (b) Acquisition with view sharing, an image is constructed after each two echoes at a rate of $20 \mathrm{~ms}$ per image 

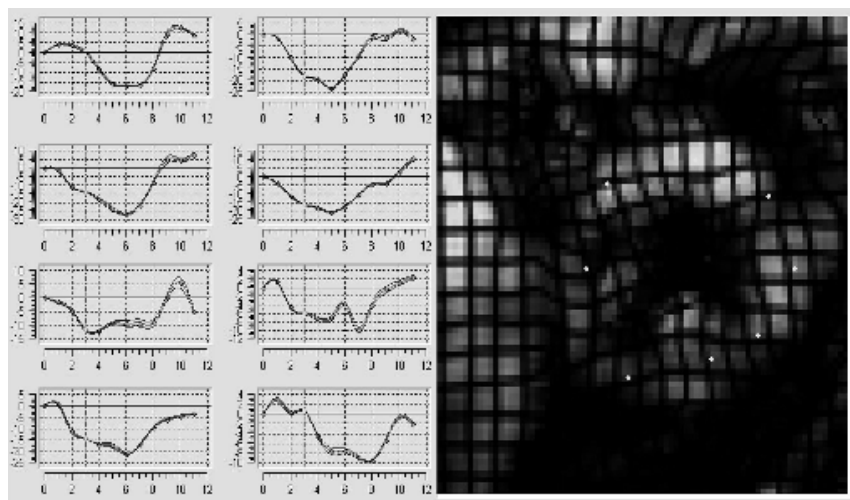

Fig. 3. A typical real-time strain analysis experiment. Right: Magnitude image of ROI is displayed with synthetic tagging and Eulerian strain superimposed. Left: The time profiles of the strain of eight material points. Horizontal axes correspond to the frame index and vertical axes correspond to strain amount

\subsection{Acquisition with Viwe Sharing}

The idea of view sharing is summarized in Fig. 2. Conventional FastHARP needs four echoes to construct an image frame as shown in Fig. 1c. A new image is created from another four echoes (see Fig. 2a.). Fig. 2b shows one possible view sharing scheme which we use in this work. $S\left[k_{x}, k_{y}\right]$ is updated every two echoes instead of four by sharing two echoes between every two consecutive images. This has the effect of improving the apparent temporal resolution.

\section{Results and Discussion}

Fig. 3. shows an example of a real-time strain analysis experiment. In this experiment, data points $S\left[k_{x}, k_{y}\right]$ are acquired by FastHARP pulse sequence, ROI image $s(n, m)$ is computed. Synthetic tagging and Eulerian strain colored map are calculated using (1) and (2), respectively and are superimposed on the magnitude image shown in Fig. 3. (right). Note the bending of the synthetic tags and the dark blue coloration in the strain maps.

Table 1. Computation time associated with each task and the total load from the three methods

\begin{tabular}{|l|l|l|c|}
\hline \multirow{2}{*}{ Task } & \multicolumn{3}{c|}{ Computation time* } \\
\cline { 2 - 4 } & zero-padding IFFT & CFT & BSI \\
\hline Harmonic image & $30 \mathrm{~ms}$ (avg.) & $3.5 \mathrm{~ms}$ (avg.) & $1.8 \mathrm{~ms}$ (avg) \\
\hline Synthetic tagging & \multicolumn{3}{|c|}{$2.2 \mathrm{~ms}$ (avg.) } \\
\hline Eulerian strain & \multicolumn{3}{|c|}{$8.5 \mathrm{~ms} \mathrm{(avg.)}$} \\
\hline Total & $14.2 \mathrm{~ms}$ & $12.5 \mathrm{~ms}$ \\
\hline Point tracking (8 Points) & $40.7 \mathrm{~ms}$ (avg.) \\
\hline
\end{tabular}

* Calculations done under Windows XP, DELL Inspiron 1.9 GHz laptop, program compiled by INTEL C++ compiler. 
It is also possible to track and plot the time profile of strain of one or more points in the myocardium in real-time. In this experiment, eight material points are selected and their strain profiles are plotted to the left. The computation times associated with each process is shown in Table 1.. Since the software runs on a multitasking operating system, specific computation times may vary; therefore, times in Table 1 . should be interpreted as estimated or representative times.

With conventional FT reconstruction, this experiment would have taken around 65 ms, which is too long for the FastHARP pulse sequence. Both CFT and BSI, however, allow this experiment to be carried out in real-time. In the future, comparison of the relative accuracies of CFT versus BSI should be carried out and attention should also be given to reduction in computation times for the point tracking and Eulerian strain computations.

Acknowledgments. This research was funded by the National Heart, Lung, and Blood Institute under Grant R01 HL47405.Notice: Jerry L. Prince is a founder of and owns stock in Diagnosoft, Inc., a company that seeks to license the HARP technology. The terms of this arrangement are being managed by the Johns Hopkins University in accordance with its conflict of interest policies

\section{References}

1. Zerhouni E. et. al.: Human heart: tagging with MR imaging: a method for noninvasive assessment of myocardial motion. Radiology 1988;169(1): 59-63

2. Osman N.F., Kerwin W.S., McVeigh E.R. and Prince J.L.: Cardiac Motion Tracking Using CINE Harmonic Phase (HARP) Magnetic Resonance Imaging. Technical Report JHU/ECE 99-3, Magnetic Resonance in Medicine, April 1999

3. Frigo M. and Johnson S. G.: FFTW: Discrete Fourier Transform (DFT) subroutine library. Cambridge, Massachusetts: The MIT, 1999, http://www.fftw.org/

4. Osman N.F., McVeigh E.R., Prince J.L.: Imaging heart motion using harmonic phase MRI. IEEE Transactions in Medical Imaging 2000;19: 186-202

5. Osman N.F., Prince J.L.: Visualizing myocardial function using HARP MRI. Physics in Medicine and Biology 2000;45: 1665-1682

6. Osman N.F. et. al. : Synthetic tagged MR images for real-time HARP imaging. Proc. ISMRM $9^{\text {th }}$ Scientific Meeting and Exhibition 2001

7. Sampath S., Derbyshire J., Osman N.F., Atalar E., and Prince J.L.: Real-time Imaging of Cardiac Strain Using Fast HARP sequence. Proc. ISMRM $9^{\text {th }}$ Scientific Meeting and Exhibition 2001

8. Kraitchman D., Sampath S., Derbyshire J.A., Heldman A.W., Prince J.L., Osman N.F.: Detecting the Onset of Ischemia Using Real-Time HARP. Proc. ISMRM $9^{\text {th }}$ Scientific Meeting and Exhibition 2001

9. Abd-Elmoniem K.Z., Sampath S., Osman N.F., Prince J.L.: Tool for automatic real-time regional cardiac function analysis using HARP. SPIE Medical Imaging 2003

10. Abd-Elmoniem K.Z., Sampath S., Osman N.F., Prince J.L.: A System for Real-Time HARP-MRI Strain Visualization. Proc. ISMRM $11^{\text {th }}$ Scientific Meeting and Exhibition 2003

11. Unser M., Aldroubi A. and Eden M.: B-Spline Signal Processing: Part II--Efficient Design and Applications. IEEE Trans. on Signal Processing, vol. 41, no. 2, pp. 834-848, 1993

12. Porat B.: A Course in Digital Signal Processing. John Wiley \& Sons, Inc. NY (1997) 